\title{
LAS ANTROPOLOGÍAS HEGEMÓNICAS Y LAS ANTROPOLOGÍAS DEL SUR: EL CASO DE ESPAÑA
}

\section{SUSANA NAROTZKY}

\section{Universitat de Barcelona}

\section{Resumen.}

El tema "Las antropologías hegemónicas y las antropologías del Sur" es una cuestión no sólo epistemológica sino de poder y de definición de los espacios posibles de producción de conocimiento antropológico. Es también un tema que entronca con cuestiones de ética y de responsabilidad y con la forma como se define la relación de la investigación antropológica con los sujetos antropológicos. Se ha debatido mucho sobre este tema, sobre todo a partir de los años 1960s, coincidiendo con los procesos de descolonización, de surgimiento de nuevos estados-nación, de nuevos discursos nacionalistas postcoloniales, de nuevos procesos de dominación, de movimientos de protesta política, civil y social en diversos lugares del mundo. Aquí voy a intentar trazar los grandes rasgos de este debate, señalando de qué manera las luchas vindicativas en determinadas coyunturas históricas han modificado el ámbito epistemológico de la antropología y transformado la relación de fuerzas entre las antropologías; pero también quiero mostrar como persisten las formas de dominación y de hegemonía de las antropologías del norte, y cómo, en el momento presente en España esta hegemonía está ligada estrechamente a la implantación del llamado proceso de Bolonia o Espacio Europeo de Educación Superior. 
Palabras clave: Conocimiento antropológico, epistemología, ética, responsabilidad, hegemonía, Antropologías del Sur.

\begin{abstract}
.
The theme "Hegemonic anthropologies and the anthropologies of the South" is both an epistemological question and an issue of power and of the definition of the possible spaces for the production of anthropological knowledge. It is also an issue that relates to the ethics and responsibilities of anthropological research towards anthropological subjects. Starting in the 1960 s and following decolonization, the emergence of new nation-states, new post-colonial nationalist discourses, new processes of domination, of social movements of politic and civic protest around the world, these issues have been widely debated. In the present contribution I want to trace the main lines of the debate, underscoring how claims and struggles in particular historical conjunctures have modified the epistemological context of anthropology and transformed the lines of power between anthropologies. I will also show the persistence of the forms of domination and hegemony of the anthropologies of the North, and how, in the present moment, in Spain, this hegemony is strongly related to the setting of the European Space for Higher Education, called the Bologna process.
\end{abstract}

Keywords: Anthropological knowledge, epistemology, ethics, responsibility, hegemony, Anthropologies of the South.

\title{
1. VARIAS TENSIONES
}

Hace más de 35 años, en 1973, en la Primera Reunión de Antropólogos Españoles realizada en Sevilla, Isidoro Moreno señalaba la doble colonización de la antropología andaluza, coincidiendo con un debate similar sobre la "descolonización" de la antropología (Stavenhagen 1971) y la importancia de la "antropología nativa” y sus aportaciones teóricas para la disciplina (Jones, 1971; Hsu, 1973) que empezaba a hacerse sentir en América Latina y en Estados Unidos, aunque menos en Europa. La primera colonización era espacial: antropólogos extranjeros, principalmente norteamericanos, concebían a España exclusivamente como un territorio lleno de informantes, como un objeto de estudio, sin otorgar nada de valor "al conocimiento de España, al progreso de la antropología española o al desarrollo de la teoría antropológica”.

La segunda colonización era teórica: una colonización por la aplicación mecánica que los antropólogos locales hacían de los conceptos y las teorías desarrolladas por los académicos angloparlantes para dar cuenta de otras realidades (Moreno, 1975: 325326). En un artículo escrito diez años después, Moreno (1984) elaboró su anterior planteamiento y trató de mostrar cómo dos etnografías muy diferentes de Andalucía, una hecha por el estructural-funcionalista inglés Julian Pitt-Rivers (1971), y la otra por un antropólogo radical estadounidense, David Gilmore (1980), sufrían ambas de formas 
flagrantes de ignorancia originadas en el conocimiento superficial de la historia local, de las realidades económicas, de los conflictos políticos y de las expresiones simbólicas de Andalucía. Moreno escribió: "En ambos estudios de comunidad, otra vez, Andalucía provee solamente el campo, y la excusa, para polémicas academicistas inútiles que tienen lugar en otros países y para obtener títulos y estatus para profesionales de la antropología que tienen poco interés verdadero en el presente y el futuro de los andaluces. Y esto tiene solamente un nombre, que es colonialismo antropológico" (Moreno, 1984: 73; énfasis en el original, subrayado mío). Su crítica subrayaba aquí la separación entre teoría y práctica, y en particular la falta de compromiso personal o político por parte del investigador extranjero. Esta realidad expresaba la reproducción de una estructura que definía lo que cuenta como conocimiento antropológico - es decir, lo que se valida como "ciencia" y lo que conforma lo que ahora llamaríamos "competencias" para adquirir el estatus profesional en el centro, pero también en los demás lugares. El resultado de esta doble colonización era por un lado la cosificación del objeto antropológico y su explotación teórica, y por otro, el estancamiento epistemológico, en la medida en que el campo del saber se convertía en un recurso económico y político controlado por una elite en los países del norte (esta elite era definida a principios de los '70 por Delmos Jones (1970) afroamericano- o Francis Hsu (1973) - sinoamericano- como el "antropólogo blanco macho").

En estas críticas tempranas apuntan ya los temas centrales de tensión entre las antropologías hegemónicas (Gran Bretaña, Francia y Estados Unidos) y las antropologías del Sur, tensiones que van a ser extremadamente enriquecedoras para el ámbito de saber antropológico tanto desde el punto de vista metodológico como epistemológico. Voy a plantear seguidamente algunas de estas tensiones.

\section{RESPONSABILIDAD Y ÉTICA ANTROPOLÓGICA}

En términos generales, el problema de la ética en la práctica antropológica se ha venido asociando con la cuestión de un compromiso político habitualmente entendido como "progresista". Un número importante de autores han planteado en determinados momentos históricos la cuestión de la tensión existente entre las responsabilidades del antropólogo/a respecto de 1) los sujetos antropológicos, 2) la disciplina como institución científica y colegiada y 3) los proveedores de fondos para investigar - públicos o privados-. Estas responsabilidades de orden distinto están en tensión permanente. Ya Katherine Gough (1968) y Gerald Berreman (1968) plantean este dilema en 1968 y lo 
resuelven dando absoluta prioridad a la responsabilidad hacia los sujetos antropológicos ${ }^{1}$. El suyo es explícitamente un planteamiento político de corte marxista. Sin embargo, ya entonces la guía de conducta que proponían Aberle, Gough, Berreman, Wolf y otros, no era evidentemente la única aceptada. Tal como nos recuerda Gough:

“En 1967 David Aberle presentó una resolución en la conferencia anual de la Asociación Americana de Antropología (AAA) que condenaba esas armas [que se usaban en Vietnam como el napalm...]. Para nuestra consternación, fue desestimada [la resolución] por la presidenta de entonces, Frederica de Laguna, y opuesta con vehemencia por Margaret Mead que argumentó que las resoluciones políticas 'no estaban en el interés profesional de los antropólogos. Hubo conmoción en la sala. David Aberle, Gerald Berreman y otros argumentaron en contra de la presidencia, pero finalmente ganamos sólo cuando Michael Harner se levantó y afirmó: 'El genocidio no está en los intereses profesionales de los antropólogos. En contra de la decisión de la presidencia, se aprobó entonces la resolución por una amplia mayoría. Fue uno de los primeros manifiestos públicos de una asociación profesional en contra de la guerra de Vietnam" (Gough, 1993).

Sin embargo, lo que me interesa subrayar de esta cita es que en aquellos años mucho/as antropólogo/as miembros de la Asociación Americana de Antropología (AAA) preferían adoptar o bien una perspectiva "profesional", respondiendo a lo que Mills (2003) ha llamado la idea de "bifurcación de valor" en la que los "hechos" son distinguibles de los "valores" y el ámbito del conocimiento debe separase del de la ética y la política; o bien una perspectiva guiada por "otra" ética ligada a la idea de "modernización" capitalista y "democratización" anticomunista en la que colaborar con el gobierno que intentaba ayudar a realizar esa utopía era una conducta ética.

Es decir, si bien el tema de la responsabilidad de los antropólogos en relación a las personas y los grupos estudiados emerge de la mano de un determinado análisis político de la realidad, existen tensiones no resueltas aún en torno a este tema: 1) el dilema perspectiva "profesional" / perspectiva "política" en antropología, y 2) el conflicto entre diversas, a veces conflictivas perspectivas "políticas" e ideas del "bien común". Aquí quiero únicamente recordar el diverso posicionamiento de los y las antropólogas

1. En dónde se asume que estos sujetos antropológicos son homogéneamente explotados/ dominados por los países capitalistas desarrollados del "centro" de los que los antropólogo/as son miembros a priori, descontando la posibilidad ya existente de antropólogos no miembros de estos centros del desarrollo capitalista, y mostrando en ese olvido el etnocentrismo anglo-céntrico de la disciplina como espacio de producción de conocimiento, incluso entre este grupo de antropólogos progresistas. También algunas antropologías "periféricas” como las de México o Brasil investigaban en el marco de formas de colonización interior (Souza Lima, 2002).

2. Esta perspectiva "profesional" es la que muchos de nuestros colegas están adoptando actualmente en relación a la implantación del proceso de Bolonia. 
españolas frente a las violencias racistas acontecidas en El Ejido en febrero de 2000: evidentemente la idea y la práctica de la responsabilidad antropológica era muy diferente para antropólogas como Emma Martín o Ubaldo Martínez y para alguien como Mikel Azurmendi, antropólogo también ${ }^{3}$.

\section{ANTROPOLOGÍA Y ANTROPOLOGÍA "NATIVA"}

El debate inicial de la responsabilidad de los antropólogos iba ligado desde el comienzo al problema de la articulación entre la práctica antropológica y la administración colonial. En el centro del debate se situaba la cuestión de la producción del sujeto "colonizable", "civilizable" o "desarrollable" por parte de una ciencia antropológica que justificaba la expansión, dominación y explotación de unas poblaciones del "Sur" por unas del "Norte". Este proceso construía conceptos útiles para la administración de poblaciones, como el de tribu, el de honor, el de área cultural, que se convirtieron en ejes de la reflexión de las antropologías hegemónicas. La crítica progresista en las antropologías hegemónicas atacó este colaboracionismo con el poder colonial desde los años '60, sin embargo no se puede decir que esto implicara el triunfo de una antropología no implicada en la transformación de la realidad. En realidad como demostraron muy pronto los críticos de la "modernización" en América Latina, la pretendida "objetividad" y "neutralidad" de algunos científicos sociales era una forma de participación muy clara en las políticas de desarrollo del neo-colonialismo. Casi siempre esta intervención vergonzante en la vida de los más débiles se ha ocultado tras un apoliticismo "profesional", ahora lo vemos con la proliferación de "expertos" que no explicitan su proyecto político, como si una ciencia social pudiera carecer de contenido ideológico. A veces se confunde la práctica reflexiva con la superación del posicionamiento social y político del antropólogo: la reflexividad sería una suerte de ascetismo profesional que habilitaría para estar por encima de la miseria del mundo y sus bajezas políticas. Pero ya Stavenhagen en 1971 subrayaba que "un componente ideológico era inseparable de la práctica profesional" de los científicos sociales y proponía la figura del científico social-activista ya que "no puede ser neutral a

3. Muchos antropólogos que no compartían las posiciones de Azurmendi reaccionaron enérgicamente. Isidoro Moreno, entonces presidente de la Federación de Asociaciones Antropológicas del Estado Español, FAAEE, con Emma Martín, escribieron un documento protestando por las posiciones de Azurmendi y cuestionando su capacidad profesional, el cual fue circulado para ser firmado por todos los demás antropólogos de la universidad. La lista de distribución original constaba de 129 antropólogos de plantilla en las universidades; 63 personas, incluyendo docentes no funcionarios, firmaron la carta — aproximadamente el 50 por ciento de la lista original-. Es difícil conocer las razones que empujaron a la gente a respaldar o no una acción corporativa de estas características, cuyo objetivo explícito era defender a la profesión antropológica contra un "cuerpo extraño" —Azurmendi-. Muchos de los que firmaron el documento no lo habrían escrito del mismo modo, pero sentían que ayudaba a poner a la antropología en un particular marco de responsabilidad. Muchos de quienes no firmaron tenían también razones corporativas, como no expresar la crítica pública sobre un colega. Otros podrían haber declinado firmar por razones pragmáticas: las agencias gubernamentales ofrecen mucha financiación para la investigación sobre inmigración. Otros respaldaban probablemente las opiniones de Azurmendi. 
las cuestiones políticas e ideológicas generales que determinan el marco de su práctica profesional" (Stavenhagen, 1971: 335).

En América Latina los y las antropólogas expresan esta participación de diversas maneras, hablan de las antropólogas como ciudadanas, como ineludiblemente partícipes en las luchas políticas en la medida en que 1) intervienen en la definición del propio campo de lo político, por ejemplo con la incorporación de la voz y la movilización de poblaciones excluidas en los estados liberales (i.e. comunidades indígenas como Yanomami), y 2) participan en los conflictos del estado-nación desde su experiencia de ciudadanas-antropólogas, con los riesgos que ello conlleva en algunos casos (Jimeno, 2004; Ramos, 2000). Esto plantea una cuestión metodológica y epistemológica central para la antropología: ¿cuál es la dimensión del "otro" que define el objeto antropológico? ¿Debemos seguir manteniendo la "alteridad" en el centro de nuestra definición de antropología como postulan algunos: es decir, debemos estudiar los procesos de producción de estructuras de alteridad (Krotz, 1997; Ribeiro, 2006: 148); o bien debemos abandonar esa "seña de identidad" y con ella la pretensión de un paradigma distintivo dentro del conjunto de las ciencias sociales?

Estas no son preguntas baladíes ni de fácil respuesta y conllevan muchas otras cuestiones fundamentales. Por ejemplo, ¿Cuál es la relación con los grupos e individuos que observamos y estudiamos? ¿Les devolvemos lo que hemos tomado de ellos? y si es así, ¿en qué forma? ¿Usurpamos sus voces para que pueblen nuestras monografías o memorias? ¿Les damos voz? O bien son ellos los que cada vez más toman la palabra, para justificarnos o contradecirnos, para utilizar nuestras palabras en sus luchas políticas. ¿Creamos la posibilidad de que emerjan polémicas con nuestros informantes, polémicas que pongan en cuestión nuestras conclusiones "científicas", nuestros conceptos, que las critiquen desde ámbitos no académicos? En definitiva, ¿cuál es el estatus de las poblaciones que estudiamos desde el punto de vista de su capacidad de producir conocimiento antropológico? ¿Cuál es su posibilidad de intervenir en el debate no sólo durante el trabajo de campo como informantes, sino en un segundo tiempo, en el debate intelectual, e intervenir no sólo sobre la fidelidad empírica sino también sobre los conceptos teóricos?

Este dilema empieza a emerger con el debate sobre la "antropología nativa" en los años 1970. En efecto lo que perciben los antropólogos "nativos" es, por un lado, su proximidad con los grupos y personas a los que observan y escuchan y con los cuales interactúan a la vez como antropólogos y conciudadanos, en su propio entorno político-social y cultural (por ejemplo la España del final del franquismo). Por otro lado, respecto a sus colegas "foráneos" los antropólogos nativos resienten ser tratados como meros "informantes", es decir situados fuera del ámbito de producción de teoría antropológica. Francis Hsu (1973) señala "los antropólogos blancos no consideran a sus colegas no-blancos como sus iguales intelectuales" (Hsu, 1973: 5) y Delmos Jones (1970) señala la incapacidad 
de la antropología "occidental" hegemónica de conversar y polemizar con teorías producidas por antropólogos "nativos". Jones apunta a la mera instrumentalización de los antropólogos "nativos" por parte de los antropólogos "reales" (hegemónicos), que los utilizan para obtener información etnográfica desde "dentro". Esta crítica temprana ya señala la exclusión de los "nativos" del campo de la producción teórica y apunta la necesidad de abrir el conocimiento antropológico a teorías formuladas desde perspectivas subalternas: "Hay antropólogos nativos [dice Jones] pero no hay antropología nativa. Lo que quiero decir es que hay poca teoría en antropología que se haya formulado desde el punto de vista de grupos tribales, campesinos o minorías. Es decir que el valor principal del investigador 'interior' no es que sus datos o sus intuiciones sobre la situación social sean mejores -sino que son diferentes. (...) [Y concluye] La emergencia de una antropología nativa es parte de una descolonización esencial del conocimiento antropológico y requiere cambios drásticos en el reclutamiento y formación de los antropólogos" (Jones, 1970: 257-258).

\section{COMUNIDADES CIENTÍFICAS DIVERSAS: ¿PARADIGMA, PARADIGMAS O MATRIZ DISCIPLINARIA?}

Esto introduce una de las tensiones más productivas del debate sobre antropologías hegemónicas y antropologías del Sur. La comunidad científica va a asumir que, en efecto, existen distintas tradiciones disciplinarias, distintas historias de la antropología marcadas por desarrollos nacionales y regionales concretos. Pero una vez se admite esa pluralización de las antropologías, ¿cómo concebir el paradigma antropológico? Es uno o es múltiple. Esto plantea una cuestión crucial desde el punto de vista epistemológico que radica en valorar la compatibilidad o conmensurabilidad de teorías antropológicas producidas desde el conocimiento situado de los y las antropólogas. Algunos antropólogos señalan que el paradigma occidental de la antropología, centrado en el estudio de la alteridad, no es el adecuado para las cuestiones que interesan a los países del Tercer Mundo en pleno proceso post-colonial y de construcción nacional. Esto lleva a algunos a proponer rupturas totales con la epistemología occidental de la Ilustración, a centrarse en paradigmas basados en marcos teóricos de saber local (por ejemplo de base teológica) que se niegan a "reconocer" a la ciencia occidental como interlocutora posible (Kaviraj, 2000; Ramanujan y Narayana Rao, en Subrahmanyam, 2000: 92; Fahim \& Helmer, 1980).

A otros les lleva a cuestionar cuál sería el nuevo paradigma antropológico en un contexto de fin del proyecto colonial que produjo el paradigma de la "alteridad". Mafeje (1976) por ejemplo, señala que el paradigma antropológico es idéntico al de las demás ciencias sociales "burguesas" - fundamentalmente positivista y funcionalista-, que está vinculado a la expansión del capitalismo liberal y llamado a desaparecer si se adopta una perspectiva epistemológica verdaderamente radical.

Otros abogan por una constante polémica en la que la confrontación de teorías que 
emergen de perspectivas diversamente situadas contribuye a la transformación del paradigma científico dentro de un marco epistemológico común. Asad (1980) por ejemplo, señala que los "paradigmas indígenas" no son necesariamente "mejores" que los Occidentales y subraya la necesidad constante de "re-trabajar críticamente lo que existe en el campo del conocimiento científico (problemas, métodos, supuestos y datos) para poder decidir aunque sea provisionalmente que un paradigma es mejor que otro". Para estos autores es esta confrontación de teorías situadas la que produce las "revoluciones científicas" de la antropología (en singular). Estas perspectivas polemicistas (o polémicas) apuntan a la creatividad de estos paradigmas alternativos que surgen fundamentalmente de las "afueras" de la antropología hegemónica pero inciden en la transformación de un único campo del conocimiento antropológico. Hsu y Textor por ejemplo, proponen (en 1978) a la Asociación Americana de Antropología organizar un "Comité de Paradigmas Alternativos" y dicen: "Nuestra actual preocupación es que la Asociación encuentre un modo de reconocer y aprovechar eficazmente su capacidad [de las minorías y de los foráneos] de romper paradigmas y de construir paradigmas, para que el campo [antropológico] como tal se beneficie" (Hsu y Textor, 1978: 12).

Esta perspectiva supone que el marco que encuadra las polémicas es único y moderno, y el objetivo es la producción de un paradigma dominante y relativamente estable durante un tiempo (Khun, 1971 [1962]).

El último desarrollo de esta tensión entre comunidades científicas hegemónicas y del Sur, heredero del post-estructuralismo, propone la "posible complementariedad relacional entre perspectivas creadas en mundos que no son en absoluto complementarios" (Cardoso de Oliveira, 2000:11). Ribeiro y Escobar hablan de "diversalidad", dicen: "Nosotros defendemos que todas las antropologías —incluyendo por supuesto a las hegemónicasson capaces de contribuir de manera dialógica en la construcción de un conocimiento más heteroglósico y transnacional" (Ribeiro y Escobar, 2008: 16). Es un intento de salvar la disciplina antropológica pero permitiendo la pluralidad de paradigmas en un marco no jerárquico. Cardoso de Oliveira habla de la "matriz disciplinaria" que comprendería una serie de paradigmas "articulados en un campo de tensión epistémica”, y este sería el aspecto diferencial de nuestra disciplina: ninguno de los paradigmas podría dominar o anular a los otros. Dentro de esta matriz la proliferación de "antropologías" es incontrolable porque el conocimiento situado sobre la realidad humana remite a infinitas perspectivas "otras" (individuales y colectivas, situadas en matrices identitarias múltiples e históricamente producidas y en relaciones de dominación cambiantes). Pero además, esa matriz disciplinaria se abre a "otros modos de hacer antropología” (Restrepo y Escobar, 2005) no exclusivamente reservados al ámbito académico o científico. El potencial revolucionario de esta nueva afirmación es enorme, pero habría que valorar en la práctica lo que esto significa, es decir, ¿qué capacidad de reconocimiento real existe para esos “otros modos de 
antropología" si no cuentan con el espacio de la antropología académica como mediador? Jean-Yves Durand (1995) relata en este sentido una experiencia interesante. En 1994 asistió a un congreso en Moscú sobre "La cultura étnica tradicional y el conocimiento folk" organizado por el Instituto de Etnología y Antropología de la Academia de Ciencias Rusa. La mayor parte de los asistentes eran rusos y además de científicos sociales había participantes de disciplinas como las ciencias naturales o la medicina. Pero además, numerosos practicantes de estos conocimientos folk -adivinación, artes marciales, prácticas de curación y de diagnóstico diversas, etc.- participaban en el congreso. La observación de Durand sobre la interacción de los académicos con estos últimos es reveladora, dice: "Mientras los participantes occidentales [académicos] se aproximaban a ellos con una actitud casi de trabajo de campo por no decir curiosidad, los científicos naturales rusos presentes estaban aparentemente bastante acostumbrados a su compañía y a colaborar con ellos y presentaron algunas comunicaciones conjuntas por ejemplo sobre la evaluación de 'un efecto extra-sensorial' (...) [Y añade:] Los académicos rusos estaban claramente interesados en integrar en la ciencia establecida a un amplio abanico de 'conocimientos folk' que incluía lo que en Occidente son dos categorías distintas, la para-ciencia y la etno-ciencia. Esta integración se llevaba a cabo desde dos enfoques distintos, el uno dedicado a demostrar el valor científico de estos asuntos, y el otro argumentando la necesidad de abrir el pensamiento científico dominante a otras formas de pensamiento y reducir el énfasis en el 'pensamiento abstracto."' (Durand, 1995: 326327). Pero al autor, sin embargo, le parece difícil establecer un proceso de comunicación real con los científicos rusos y sus colegas "Fol." sin proceder antes a la clarificación de los términos y conceptos que se emplean en estos diversos ámbitos de conocimiento. Ahora bien, una se pregunta hasta qué punto esa estabilización conceptual que permitiría la comunicación no comporta de entrada un sometimiento a nociones de categorías reificadas y abstractas propias de la ciencia occidental.

\section{LA CAPACIDAD TRANSFORMADORA DE LA ANTROPOLOGÍA}

Entre la disolución del paradigma antropológico (Mafeje, 1998) y una matriz disciplinaria sin límites de "Otras antropologías y antropologías de otro modo" (Restrepo y Escobar, 2005) se encuentra la cuestión de la pertinencia de una definición centrada en la "otredad". La tensión remite aquí a lo que esto significa y posibilita para transformar la sociedad en distintos contextos históricos. Para Mafeje (2002: 8-10) y otros científicos sociales africanos, el nuevo nacionalismo progresista africano ${ }^{4}$ representa una lucha política anti-imperialista y una lucha por la igualdad social y la dignidad humanas que pasa por la desaparición del paradigma de la "alteridad" que caracterizó la fundación

4. Este nuevo nacionalismo se presenta como crítico a la vez del estado neo-colonial y de los experimentos socialistas africanos. 
de la antropología (esa "ranura salvaje" [savage slot] de la que habla Trouillot, 1991). En palabras de Shivji (2003) "La quintaesencia del nacionalismo era y es el antiimperialismo. Era una demanda de lucha contra, más que por, algo. Era una expresión de la lucha contra la negación — negación de humanidad, negación de respeto y dignidad, negación de la africanidad del africano." En esta lucha política el referente de la igualdad social se erige para estos intelectuales como el fundamento de una transformación en profundidad de las relaciones sociales locales, regionales, nacionales y transnacionales y busca anular la significación de la diferencia. Contra el estado neo-colonial y los fallidos experimentos del socialismo real africano, proponen un nuevo nacionalismo regional en un estado descentralizado, que permita plantear los problemas y las soluciones desde la proximidad, y desde una nueva democracia que definen como "socialismo democrático" (Mafeje, 2002; Shivji, 2003).

Sin embargo, otros desarrollos históricos producen otros intelectuales orgánicos y otras formas de lucha. En América Latina, Carmen Martínez (2007) y Carlos de la Torre (2007) estudian la dimensión política de los movimientos indígenas y afro-americanos subrayando su constitución como interlocutores de un discurso y una práctica corporativista del Estado. Señalan para el caso de Ecuador la articulación entre formas débiles de ciudadanía, movimientos indígenas y lógicas corporativistas o personalizadas de movilización política de los grupos subalternos. En un proceso histórico en que la igualdad de derechos de la ciudadanía liberal no se logra implantar en la cotidianidad, en la que subsisten esos grupos excluidos (afrodescendientes, indígenas), una de las estrategias de los diversos agentes sociales (tanto dominantes como dominados) ha sido el recurso corporativista (de la Torre, 2007: 162-70). Este proceso puede entenderse como una expresión de ese desplazamiento de la administración de poblaciones hacia el ámbito de las instituciones no estatales. El Estado regula el conflicto en la sociedad a través de los representantes de los diversos grupos de interés que componen el "cuerpo" social. Esta estructura, producto en parte de la historia colonial en América (Clavero, 1994) y de los avatares modernizadores del largo siglo diecinueve, se va a expresar de una manera particularmente vívida en la consolidación de los movimientos indígenas y afroecuatorianos. Instituciones muy diversas (Banco Mundial, misioneros salesianos y combonianos, agencias de desarrollo variadas) intervienen en la creación de unos interlocutores definidos en términos de identidad cultural (indígena, afroecuatoriana), que se posicionan de este modo en unos ámbitos de enunciación particulares para reclamar y acceder a determinados recursos y enfrentarse a su situación. Pero Carlos de la Torre apunta que la utilización de discursos y prácticas corporativistas no excluye el recurso al discurso de la igualdad y la ciudadanía (2007: 173) por parte de las poblaciones subalternas. En estas coyunturas no es de extrañar que emerjan entre los antropólogos propuestas mucho más centradas en las "estructuras de la alteridad" y la significación política de la diferencia. 
Estos dos ejemplos también nos muestran de qué modo nuestra disciplina, la antropología, en su construcción diversa y polémica, es fruto de procesos históricos localizados y eminentemente politizados. Es esta dimensión política e histórica la que nos orienta en nuestra reflexión epistémica. Todos somos, ineludiblemente antropólogos nativos y antropólogas ciudadanas y, como decía Stavenhagen, no existe investigador neutral ni paradigma que no refleje una toma de posición respecto a la realidad del mundo. Los y las antropólogas toman posición con su análisis de la realidad y se someten al juicio crítico, científico y político, tanto de los sujetos sociales a los que han hecho objeto de su observación y análisis, como de los y las colegas de la academia, como de la ciudadanía en general. Entre el colapsamiento de la "otredad" y la proliferación infinita y homogénea de "alteridades" queda el espacio de una práctica científica antropológica que se reconoce como ineludiblemente política, a la vez "otra" y "única" puesto que propone un modelo de transformación social, pero un modelo situado.

\section{CONCLUSIÓN: LAS ANTROPOLOGÍAS DEL SUR Y EL PROCESO DE BOLONIA}

La lucha por el reconocimiento de las antropologías del Sur y de sus propuestas y paradigmas diversos, requiere un espacio de posibilidad, requiere que exista un espacio crítico, un espacio de polémica en el que poder disentir y construir nuevos modelos. Si ese espacio desaparece en las instituciones universitarias, tendremos que luchar por crearlo en otro sitio, nos convertiremos en disidentes y resistentes. Como decían Hsu y Textor (1978) los paradigmas rompedores e innovadores vienen de las "afueras" del conocimiento instituido, pero también de las "afueras" de los grupos que dominan la producción de conocimiento (como señala el famoso aforismo de Koselleck "Si la historia a corto plazo la hacen los vencedores, históricamente las ganancias en conocimiento provienen a la larga de los vencidos" (2001: 83).

Hace tiempo que las reformas neo-liberales del Estado intentan transformar la producción de conocimiento a través de la financiación de la investigación mediante la propuesta de líneas prioritarias, acciones estratégicas, colaboración con las empresas, etc. Esta revolución silenciosa ha supuesto para muchos la necesidad de plegarse a determinados conceptos, a determinadas problemáticas formuladas según pautas predefinidas por intereses político-económicos particulares. Hemos resistido mediante la picaresca, nos adaptamos a la norma impuesta para solicitar el proyecto y luego "hacemos lo que queremos". Pero, poco a poco, acabamos queriendo hacer lo que esperan que hagamos, acabamos utilizando los conceptos y métodos que sabemos que tienen mayor aceptación y "productividad" en las instituciones que controlan la financiación pública y privada de investigación actualmente: por ejemplo, pocos antropólogos se atreven a proponer un trabajo de campo prolongado en un proyecto $\mathrm{I}+\mathrm{D}$, y muchos es porque creen que "ya" no es necesario. Casi ninguno se plantea aprender la lengua de los inmigrantes a los que va estudiar en España cuando es distinta de la propia (p.ej. Rumano, Chino o 
Wolof), evidentemente ninguna entidad financiaría ese aprendizaje como parte de una investigación pero tampoco lo consideraría un requisito imprescindible... y el investigador acaba también creyendo que se las podrá apañar con el castellano y quizá el inglés. Ni siquiera nos paramos a pensar lo que eso significa como sesgo inicial para nuestras etnografías, mucho menos lo que significa como posicionamiento político de nuestra metodología.

El proceso de Bolonia es la implantación de la hegemonía neoliberal anglosajona (modelo iniciado por Thatcher y Reagan en los '80) en el ámbito universitario, tradicionalmente el ámbito del saber. Busca la sumisión de la producción de conocimiento al principio de utilidad marginal que rige el modelo neoclásico de la economía capitalista: conceptos como "eficiencia", "productividad", "competitividad", "gestión", etc. se consideran ahora como los más idóneos para orientar la producción de conocimiento. Evidentemente, el pensamiento crítico que duda y busca permanentemente no responde al principio de maximización de la "utilidad". La "profesionalización" de las carreras en los nuevos grados es, para las ciencias sociales, el establecimiento de esa ideología de la "neutralidad" científica cuyos resultados nefastos para grandes porciones de la humanidad a menudo hemos documentado y analizado en nuestro trabajo (no hay más que ver los proyectos de desarrollo del Banco Mundial guiados por la teoría de la modernización). Una "neutralidad" que tiene nombre y apellido político: hegemonía neoliberal. La sumisión a la implantación del proceso de Bolonia supone aceptar una forma muy particular de producción de conocimiento, la de los vencedores. Si aceptamos colaborar quizá logremos que nos publiquen más artículos en revistas de impacto (casi siempre anglosajonas), pero es muy probable que esos artículos no sean muy distintos de los otros que publican esas revistas, es probable que adopten objetos de estudio similares (de moda), metodologías y marcos teóricos hegemónicos. La sumisión a la hegemonía del mercado en la educación es la sumisión a un oscurantismo economicista muy distante y distinto de un proyecto progresista de transformación social. Es también el fin del pensamiento crítico, reflexivo y político en las ciencias sociales, quizás el mejor legado de la Ilustración. Como antropólogos y antropólogas tenemos la responsabilidad de saber analizar este proceso y entender cuál es su orientación político-económica más allá de las retóricas de propaganda ideológica del poder político y económico. Luego, cada cual tomará partido según su conciencia. Personalmente creo, como Rodolfo Stavenhagen señalaba en 1971, que: "Tenemos la responsabilidad de ayudar a promover sistemas educativos para la liberación del ser humano y no para su domesticación y sometimiento a los sistemas establecidos de dominación". 


\section{REFERENCIAS BIBLIOGRÁFICAS}

ASAD, T. (1980) Comment to Fahim, H. y Helmer, K. (1980) "Indigenous Anthropology in Non-Western Countries: A Further Elaboration". Current Anthropology Vol.21(5), pp. 661-662.

BERREMAN, G. (1968) "Is Anthropology Alive? Social Responsibility in Social Anthropology". Current Anthropology, 9(5), pp. 391-396.

Cardoso de Oliveira, R. (2000) "Peripheral anthropologies versus central anthropologies". Journal of Latin American Anthropology 4(2)-5(1), pp. 10-30.

DE LA TORRE, C. (2007) “Entre el corporativismo y las ciudadanías débiles”. En Bretón, García, Jové y Vilalta (Eds.) Ciudadanía y exclusión: Ecuador y España frente al espejo. Madrid: Libros de la Catarata.

DURAND, J-Y (1995) “'Traditional Culture' and 'Folk Knowledge': Whither the Dialogue between Western and Post-Soviet Anthropology?". Current Anthropology Vol.36(2), pp. 326-330.

FAHIM, H. y HELMER, K. (1980) "Indigenous Anthropology in Non-Western Countries: A Further Elaboration”. Current Anthropology Vol.21(5), pp. 664-653.

GILMORE, D. (1980) The People of the Plain. Class and Community in Lower Andalusia. New York: Columbia University Press.

GOUGH, K. (1968) “New Proposals for Anthropologists”. Current Anthropology, 9(5), pp. 403-407.

GOUGH, K. (1993) “'Anthroplogy and Imperialism’ Revisited”. Anthropologica, Vol.35 (1).

HSU, F. (1973) "Prejudice and Its Intellectual Effect in American Anthropology: An Ethnographic Report”. American Anthropologist, Vol.75(1), pp. 1-19.

HSU, F. y TEXTOR, R. (1978) “Third World Anthropologists and the Reappraisal of Anthropological Paradigms”. Anthropology Newsletter, Vol.19(6), pp. 11-12.

JIMENO, M. (2004) "La vocación crítica de la antropología latinoamericana”. Maguaré 18 , pp. 33-58.

JONES, D. (1971) “Towards a Native Anthropology”. Human Organization, Vol.29(4), pp. 251-259.

KAVIRAJ, S. (2000) “Des avantages dêtre un barbare.” L'Homme 156:, pp.75-86.

KHUN, T.S. (1971 [1962]) La estructura de las revoluciones científicas. México: Fondo de Cultura Económica. 
KOSELLECK, R. (2001) Los estratos del tiempo: estudios sobre la historia. Barcelona: Paidós.

KROTZ, E. (1997) "Anthropologies of the South. Their rise, their silencing, their characteristics". Critique of Anthropology Vol.17(3), pp.237-251

MAFEJE, A. (1976) “The Problem of Anthropology in Historical Perspective: An Inquiry into the Growth of the Social Sciences". Canadian Journal of African Studies, Vol.10(2), pp. 307-333.

MAFEJE, A. (1998) "Anthropology and Independent Africans: Suicide or End of an Era?”. African Sociological Review Vol.2(1), pp. 1-43.

MAFEJE, A. (2002) "Democratic governance and new democracy in Africa: agenda for the future" African Forum for Envisioning Africa to be held in Nairobi, Kenya, 26 - 29 April.

MARTINEZ, C. (2007) “ ¿Es multiclturalismo estatal un factor de profundización de la democracia en América Latina?: Una reflexión desde la etnografía sobre los casos de México y Ecuador”. En Bretón, García, Jové y Vilalta (Eds.) Ciudadanía y exclusión: Ecuador y España frente al espejo. Madrid: Libros de la Catarata.

MILLS, D. (2003) “'Like a horse in blinkers'?: a political history of anthropology's research ethics". En Caplan, P. (Ed.) The ethics of anthropology: debates and dilemmas. London: Routledge.

MORENO, I. (1975) “La investigación antropológica en España." en Primera reunión de antropólogos españoles, compilado por A. Jimenez, 325-333. Sevilla: Universidad de Sevilla.

MORENO, I. (1984) "La doble colonització de l'antropologia andalusa i perspectives de futur.” Quaderns de l'ICA, no. 5, pp. 69-84.

PITT-RIVERS, J. (1971 [1954]) The People of the Sierra, 2nd ed. Chicago: University of Chicago Press.

RAMOS, A.R. (2000) "Anthropologist as political actor: between activism and suspicion" Journal of Latin American Anthropology 4(2)-5(1): 172-189

Restrepo, E. y Escobar, A. (2005) “'Other Anthropologies and Anthropology Otherwise’ Steps to a World Anthropologies Framework" Critique of Anthropology Vol.25(2): 99129

RIBEIRO, G. Lins (2006) "Antropologias mundiais. Para um novo cenário global na antropologia”. Revista Brasileira de Ciências Sociais Vol. 21(60), pp. 147-184. 
RIBEIRO, G. Lins y ESCOBAR, A. (2008) "Prefacio. Antropologías del mundo: transformaciones disciplinarias dentro de sistemas de poder" en Ribeiro y Escobar (eds.) Antropologías del mundo. Transformaciones disciplinarias dentro de sistemas de poder. Popayán: Envión editores / CIESAS/ Wenner Gren.

SHIVJI, I.G. (2003) "The rise, the fall and the insurrection of nationalism in Africa". Keynote Address to the CODESRIA East African Regional Conference held in Addis Ababa, Ethiopia, October 29-31.

STAVENHAGEN, R. (1971) “Decolonizing Applied Social Sciences". Human Organization Vol.30 (4), pp. 333-357.

SUBRAHMANYAM, S. (2000). "Parler pour autrui” L’Homme 156, pp. 87-98.

TROUILLOT, M-R. (1991) "Anthropology and the Savage Slot: The Poetics and Politics of Otherness", en Fox, R. (Ed) Recapturing Anthropology. Working in the Present. Santa Fe, New Mexico: School of American Research. 